\title{
The Influence of Family Context on Identity Processing
}

\author{
Joaquín Ibáñez-Alfonso, ${ }^{1,2,3}$ Rong Wei Sun, ${ }^{1}$ and Gertina J. van Schalkwyk ${ }^{1}$ \\ 1 Department of Psychology, University of Macau, Taipa, Macau, China \\ 2 Department of Developmental and Educational Psychology, University of Seville, Spain \\ 3 Department of Psychology, Universidad Loyola Andalucía, Spain
}

\begin{abstract}
Tre he purpose of this project was to investigate the role of perceived parenting styles in the choice of identity processing and commitment among a group of Chinese youth. Examining the identity processing styles of 209 young people using the ISI-4 (Smits et al., 2008) and Parental Authority Questionnaire (PAQ; Buri, 1991), we hypothesised that parental styles and family socio-economic status would differentially impact the identity commitment of youth in Macao. The findings corroborated Berzonsky's (2004) model, with minor differences, showing a positive relation between authoritative parenting and informational processing and identity commitment. However, a negative relation between a normative processing style and identity commitment was found in the Chinese sample. Testing for the moderating effect of socioeconomic status showed that authoritative parenting combined with high SES levels lead to the informational processing style.
\end{abstract}

Keywords: diffuse-avoidant processing, identity styles, informational processing, normative processing, parenting, socio-economic status

Young people are in the process of identity commitment, and in their 'struggle to negotiate the competing information of an interior and external world' (Singer, 1995, p. 430), they are searching for individuation and a sense of self in the world. This is also evident for young people in Macau, a unique territory with a long history of East-West intermingling and posing many challenges to the young person growing up in the territory (Xu, 2011). On one hand, traditional Chinese values and parenting styles prevail, while on the other hand, young people have regular interaction with non-Chinese values and practices through an education system - specifically, tertiary education - that is modelled on international standards (Yeh \& Bedford, 2003). There is a complex blend of thought processes that emerge as young people increasingly connect to one another in ecological, economic, political, and social ways, so that the traditional perspective of cultural differences no longer holds true (Barcinski \& Kalia, 2005; $\mathrm{Xu}, 2011$ ). Extensive direct (e.g., the university environment comprised of foreign teachers, exchange students and peers from the mainland) and indirect (e.g., the internet and other media) exposure contributes, for example, to the possibility of confusion in the mediated action of ideological becoming (Tappan, 2005). However, little is known about the identity development and commitment of young people in Macau.

Despite changes to the notion of family and parenting in the 20th century, the Chinese family living in Macau still maintains a great sense of connectedness with past generations (Bond, 1991; Chang, McBride-Chang, Stewart, $\& \mathrm{Au}, 2003)$, with strong family values and a fundamental belief in family and kinship (Georgas, 2003; Yeh \& Bedford, 2003). As a context primarily oriented towards relationship and harmony, family members solicit each other's attention, approval and support, and respond to each other's needs, expectations and distress, thus co-creating family as uniquely interdependent and relational (Low \& Stocker, 2005). Georgas (2003) and others (e.g., Kagitcibasi, 2002) note the essential differences in structure and functioning of the family, and variances in belief systems, child rearing practices, and parenting styles between cultures. Among Chinese parents, parental authority is evident in a punitive approach to child rearing and the establishment of moral behaviour, and the parental styles employed towards their adolescent child who is in the process of identity development (Chang, Schwartz, Dodge, \& McBrideChang, 2003). Within this context, the question is to what extent the parenting styles of Macau parents and tadolescents' perceptions of demandingness and responsiveness of their parents would influence the identity processing styles they adopt during late adolescence.

\section{Identity Processing Styles}

In Erikson's (1968) psychosocial theory, the major developmental task of youth during their adolescent years is

Address for correspondence: Gertina J. van Schalkwyk, Department of Psychology, University of Macau, Macao (SAR), China. Email: givsumac@gmail.com 
to form a stable and coherent self-identity. Self-identity provides the adolescent with a subjective sense of inner wholeness and helps them to answer the questions about purpose and meaning of life. Adolescents construct their identities in the context of the environment around them, the interactions with society, and also the transitional roles of their age group (Erikson, 1968; Singer, 1995; Thorne, 2004). Identity commitment in late adolescence represents the integrative framework within the self that emerges during the transition to adulthood. Therefore, identity can be seen as a product of negotiation with the surrounding culture. In the ongoing process of dialoguing between the individual and the social-cultural structures of family and society, the adolescent explores her/his identity, finding a unique sense of self.

In order to account for individual variation in identity development, Berzonsky $(1989,1990)$ proposed a model related to the social-cognitive processes that adolescents use to construct identity. In this model, Berzonsky proposes three identity processing styles, namely informational, normative, and diffuse-avoidant, and claims that individuals use varying processing styles as they 'process self-relevant information, negotiate identity issues, and make decisions' (Berzonsky, 2004, p. 213). The young person who uses the informational processing style will actively seek and evaluate relevant information before making decisions. These individuals are self-reflective, conscientious, and problem-focused (Berzonsky, 1990; Berzonsky \& Ferrari, 1996), and in late adolescence they have achieved or are in the process of forming an identity commitment (Berzonsky, 1989, 1990; Berzonsky \& Kuk, 2000). Individuals who tend to conform to standards and social norms usually employ the normative processing style, forming identity commitments without an active process of self-exploration. They rely automatically on the expectations of authority figures and significant others, adopt a collective self-definition, and have a high need for structure and cognitive closure (Berzonsky, Nurmi, Kinney, \& Tammi, 1999). The diffuse-avoidant processing style is evident among adolescents who delay, procrastinate, and try to avoid conflict resolution, and who are reluctant to face identity issues. They are uncommitted and present a diffused identity status (Berzonsky \& Neimeyer, 1994; Streitmatter, 1993). According to Berzonsky's social-cognitive model, the late adolescent is assumed to be capable of using all three processing styles, and Berzonsky and Ferrari (1996) claim that situational factors, contextual demands, and personal preferences influence the style that the adolescent will employ in his/her identity development.

Previous research has also investigated the role of parental authority patterns on identity processing styles and identity commitment (Berzonsky, 2004). Family, and in particular, the parents and their caregiving styles, have a great influence on an individual's feelings, thoughts and actions, as well as the variation of identity commitment in late adolescence. With family authority accounting for $50 \%$ of the variation in strength of identity commitment,
Berzonsky (2004) indicated that identity processing styles are greatly mediated by the relationship between parental authority and identity commitment. This is consistent with the view that the family authority patterns may influence the adolescent when constructing a sense of identity (e.g., Chang, McBride-Chang et al., 2003; Chang, Schwartz et al., 2003). Although the literature has shown the relation between parental authority patterns and identity styles, most of the previous studies have been carried out in Western contexts. The present study aimed to investigate whether similar results could be found in an Asian context such as Macau.

In the authoritative, authoritarian, and permissive parenting styles, varying degrees of authority emerge, depending on the degree of control or demandingness exerted over a child's behaviour and the warmth or responsiveness with which a parent responds to a child's needs both physical and psychological (Barnes, Brown, McDermott, Bryant, \& Komrey, 2012; Baumrind, 1991; Lawford, Pratt, \& Hunsberger, 2005). A characteristic of authoritative parenting is the involved and supportive nature with which parents engage with their children, despite the high degree of control over their behaviour (Gauvain \& Huard, 1999; Zhou, Eisenberg, \& Wang, 2004). While respecting the child's search for autonomy and the freedom to make her/his own decision, authoritative parents set reasonable limits on the child's behaviour and use verbal communication to guide the child towards identity commitment in late adolescence. Parents encourage independence and are considered both highly demanding and highly responsive. On the other hand, authoritarian parents are highly demanding and set absolute standards. These parents also tend to use coercive control, often expecting absolute obedience from their children. These parents are less responsive to the child's needs and discourage verbal give and take (Barnes et al., 2012). Maccoby and Martin (1983) and Darling and Steinberg (1993) distinguish between indulgent (or permissive) parents and neglecting parents. Indulgent/permissive parents are typically low in demandingness but high in responsiveness. They do not set strict rules or expect obedience to external standards, but rather act in a non-restrictive way and have good communication that is accepting and affirming of the adolescent's identity development process. 'The parent is a resource for the child but does not view himself or herself as an active agent in the shaping of the child's behaviours' (Lerner \& Castellino, 2000, p. 48). Least demanding and responsive is the neglecting parenting style, which sets few rules for behaviour, is less responsive, provides little or no support, and allows unlimited freedom to the child for self-regulation.

Further to parenting styles, Lawford and colleagues (2005) suggest that the combination of warmth (responsiveness) and control of behaviour (demandingness) facilitate social responsibility in children in support of their identity development. With a high degree of control and responsiveness, authoritative parenting positively 
correlates with a positive self-image and self-acceptance, flexibility, and the child being able to accept responsibility for her/his actions (Jackson, Pratt, \& Hunsberger, 2005; Trevatt, 2005). Authoritative parenting is also associated with less risk-taking behaviour, healthy exploration of multiple options, and a more informational processing style (Berzonsky, 2004). In contrast, authoritarian parenting is often characterised by less warmth and either high or low demandingness. This leads to a lower self-image and less self-acceptance, and children growing up with authoritarian parenting often enter late adolescence with less self-regulation capability and a foreclosed identity that allows little room for exploration, with a commitment to the norms set by the parents. Berzonsky (2004) claims that these young peope tend to choose the normative processing style, although positive perceptions of the authoritarian parental control would assist with choosing the informational processing style. Permissive parenting encourages greater flexibility, but can lead to the adolescent becoming inclined towards high-risk behaviour, substance abuse/misuse, and little pro-social engagement in society (Bednar \& Fisher, 2003). The child of permissive parents also experiences a higher degree of role confusion in late adolescence, and employs the diffuse-avoidant processing style, often taking longer than her/his counterparts from authoritative and authoritarian parents to commit to a particular identity style (Jackson et al., 2005; Lerner \& Castellino, 2000).

\section{Family Socio-Economic Status}

Family socioeconomic status (SES) is a multidimensional construct that combines different social and economic measures and allows for the characterisation and comparison (Hollingshead, 1975) of the family context in which the adolescent constructs identity. The socioeconomic measures consistently accepted by the majority of researchers include income, education, and occupation. These three measures generally show very high correlations, allowing integration into a single multidimensional index (McLoyd, 1998). There is a broad consensus in the scientific community regarding the potential negative effects of socioeconomically depressed contexts on cognitive development in childhood (e.g., Bradley \& Corwyn, 2002; Hackman \& Farah, 2009; Thomas, Forrester, \& Ronald, 2013). These effects are particularly appreciable in language and executive functions development, and have been corroborated not only through behavioural performance tests but also through direct measures of brain structure and functionality (e.g., Farah et al., 2006; Hanson et al., 2013; Kishiyama, Boice, Jimenez, Perry, \& Knight, 2009).

Following the bio-ecological model of human development (Bronfenbrenner \& Ceci, 1994), we can organise some of the major risk factors related to SES that influence the development of identity in adolescence. These risk factors can be considered as biological (e.g., child malnutrition, inadequate medical care, perinatal exposure to toxic substances), psychological (e.g., increased exposure to stress and violence, and inappropriate parenting styles and speech stimulation), and environmental (e.g., less safe and stimulating neighborhoods, or schools with fewer resources), all of them interacting with the socioeconomic characteristics of the family in building the developmental context for the adolescent (American Psychological Association Task Force on Socioeconomic Status, 2007; Letourneau, Duffett-Leger, Levac, Watson, \& Young-Morris, 2013). Currently there is not enough evidence supporting that family SES influences perceived parenting styles, although some researchers claim that SES can have a significant impact on the academic achievement of adolescents (e.g., Hoff, Laursen, \& Tardif, 2002; Leung, Lau, \& Lam, 1998; Spera, 2005). However, the influence of SES on identity processing styles has not been studied before. Insofar as SES might be important source materials for developing identity, it is feasible to expect an impact of family SES on the relationship between perceived parenting and the different identity processing styles that youth commit to in their late adolescence.

Leung and $\mathrm{Xu}$ (2013) claim that the socioeconomic status of the families of children and youth in Macau is correlated with vigour, self-esteem, and mood states, and positively correlated with the youths' relationship with their parents. Additionally, many researchers (e.g., Schady, 2011; Fernald, Marchman, \& Weisleder, 2013) found that the parents' education, which is a part of the measure of SES, will influence the cognitive functioning of their children. It is thus possible to assume that socioeconomic status might influence the identity processing styles of adolescents, especially the informational. Youth adopting the informational processing style are more likely to seek and evaluate relevant information compared with other identity processing styles, and such information would most likely be more forthcoming in families with a high SES. However, there has been little study conducted to explore the relationship. Therefore, in this study we also investigated the SES of Macau families, anticipating that SES would moderate the relation between authoritative parenting style and informational identity processing.

\section{Method}

The present study examined the identity processing styles, perceived parenting styles, and socioeconomic status of male and female youth in Macau studying at a local tertiary institution. With little known about the identity development of Chinese youth, the major purpose of this project was to examine whether Berzonsky's model (2004) could be applied to and would fit the identity processing styles of male and female college students in Macau (see Figure 1). Consistent with Berzonsky's model, we hypothesised a relationship between identity processing styles (i.e., informational, normative, and diffuse-avoidant) and parental styles, and the contribution of each processing style to 


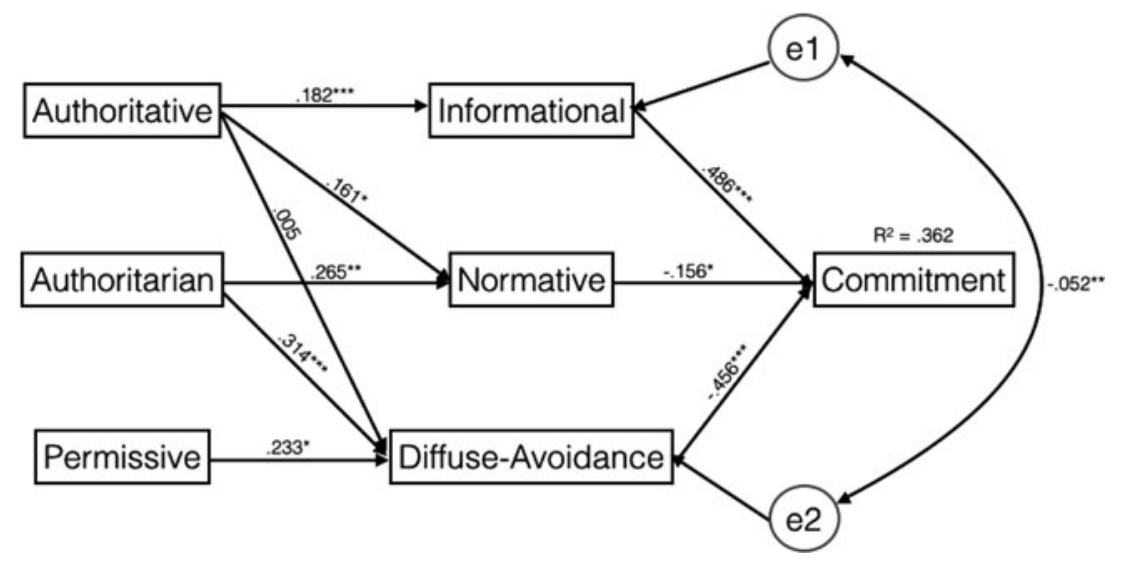

\section{Figure 1}

Model 1: The path model of parental authority and identity processing styles predicting commitment suggested by Berzonsky (2004). Note: ${ }^{*} p<.05,{ }^{* *} p<.01,{ }^{* * *} p<.001$.

identity commitment. In addition, we hypothesised that identity processing styles would mediate the relationship between perceived parental authority and commitment. We further hypothesised that SES would moderate the relationship between the perceived authoritative parenting style and informational identity processing, but only if the family had a high SES.

\section{Participants}

Participants for this study were recruited using a system employed by the psychology department requiring all students in an undergraduate psychology course to engage in a research project of their choice for credit. Students could choose from a range of research projects. A total of 209 students ( 134 women, 75 men, $M_{\text {age }}=19.3$ years, age range 17-25 years) were recruited for this study through purposive sampling. The majority of students were Chinese (97\%) from diverse socioeconomic backgrounds, and $76 \%$ were in the first year of their degree program. All the participants had sufficient language proficiency to comprehend the survey questionnaire presented in English and Chinese. Ethical approval was obtained from the department, and informed consent was obtained from the students prior to completing the survey, which assured them of confidentiality and anonymity and their right to withdraw without penalty - that is, the student still received credit for participation although he/she did not complete the survey. No coercion was involved.

\section{Instruments}

A survey questionnaire was designed in English and Chinese comprising demographic information, the Identity Style Inventory version 4 (ISI-4; Smits et al., 2008) and the Parental Authority Questionnaire (PAQ; Buri, 1991). Both the ISI-4 and the PAQ were not available in Chinese, and prior to conducting the study, the question statements in both instruments were translated for convenience of participants whose native language was mainly Chinese.
Translation experts familiar with both English and Chinese as well as psychological research assisted with the translation, and back translation was conducted to confirm that all statements were as close as possible to the original. The translated version used simplified Chinese, which is the same for both dialects (i.e., Cantonese and Mandarin) although meanings can differ somewhat. Where relevant, slight adjustments were made to statements to ensure the coverage of cultural variations. Finally, bilingual versions (English-Chinese) of both questionnaires were administered to our participants.

Identity styles and commitment. The revised Identity Style Inventory version 4 (ISI-4) was used to test participants' identity processing styles (Smits et al., 2008). This version has 34 items forming three scales for informational processing style (seven items; e.g., 'When facing a life decision, I take into account different points of view before making a choice'; Cronbach's $\alpha=.72$ ), normative processing style (seven items; e.g., 'I automatically adopt and follow the values I was brought up with'; Cronbach's $\alpha=.65$ ), and diffuse-avoidant processing style (eight items; e.g., 'I am not really thinking about my future now; it is still a long way off'; Cronbach's $\alpha=.65$ ), and an additional scale for identity commitment (10 items; e.g., 'I know what I want to do with my future' Cronbach's $\alpha=$ .83). Participants responded to the items using a 5-point scale ranging from 1 (not at all like me) to 5 (very much like me). According to Smits et al. (2008), the ISI-4 scales had adequate internal and test-retest reliabilities, as well as satisfactory convergent validity with other measures with identity status or identity content emphasis. Item 31, 'When others say something that challenges my personal values or beliefs, I automatically disregard what they have to say', and item 22, 'I try not to think about or deal with personal problems as long as I can', were removed from the final data set due to low post-hoc reliability scores.

Perceived parenting style. The Parental Authority Questionnaire (PAQ; Buri, 1991) was used to explore the 
youths' perceptions of parenting styles for both parents. The PAQ included 30 items per parent on two dimensions of demandingness and responsiveness for each of the permissive, authoritarian, and authoritative parental prototypes, and provided a score for both the mother and the father independently. Sample items included 'As I was growing up, my parents seldom gave me expectations and guidelines for my behaviour' (permissive; 10 items), 'As I was growing up my parents did not allow me to question any decision they had made' (authoritarian; 10 items), and 'As I was growing up I knew that what my parents expected of me in my family, but I also felt free to discuss those expectations with my parents which I felt that they were unreasonable' (authoritative, 10 items). Participants responded to each item using a 5-point scale ranging from 1 (strongly disagree) to 5 (strongly agree). A cumulative score for the family was extracted combining the scores for the mother and the father. If one of the parents was absent, the score of the present parent was considered to be the family score.

Demographic and socioeconomic questionnaire. Also included was a demographic questionnaire, including queries regarding ethnicity, language spoken at home, and current academic status of the participant. With regard to the family's socioeconomic status, the demographic questionnaire also included questions concerning parents' highest academic degree, occupation, income, and the family structure.

\section{Analysis}

The researchers conducted both exploratory and descriptive analyses using the statistical package SPSS version 20. Path analysis was carried out using $\mathrm{R}$ script ( $\mathrm{R}$ Core Team, 2014). A first analysis was done to test the normality assumptions of all the variables - that is, the identity processing styles and commitment measure (ISI-4), the perceived parenting styles (PAQ), and the socioeconomic status (SES) of the family. The measures were all normal, except for the normative identity processing style, which we corrected using a correction factor proposed by Satorra and Bentler (1994). The correction factor ensured both maximum-likelihood and robust analysis for the ensuing path analysis to avoid violation that would influence the results. Standard model fit indices were used to further test the fit of Berzonsky's (2004) model (Beaujean, 2014; West, Taylor, \& Wu, 2012). The incremental indices: CFI (>.95) and TLI (>.95), parsimony indices: AIC, BIC (smaller indicated better), and RMSEA $(<.06)$, absolute fit indices: SRMR $(<.08)$, as well as the non-significant chi-square value were calculated to determine whether Berzonsky's model could apply to Chinese participants. If most indices met the standards above, we could assume a fit. However, if most indices did not meet the standard, we could drop some paths in order to suggest a model for Chinese participants that would fit better. The two models were compared using chi-square test, and a $p$ value larger than .05 indicated that the revised model fit the data better. The sample size $(N=209)$ was considered adequate for the analyses, ensuring satisfactory statistical power (Bentler \& Chou, 1987; Kline, 2005; Quintana \& Maxwell, 1999).

In order to test the hypothesis that socioeconomic status (SES) moderated the relation between authoritative parenting style and informational processing style, the researchers derived a component measure of SES for the family based on the mean parental educational level, the mean parental occupational status, and the family incometo-need ratio (McLoyd, 1998; Noble, McCandliss, \& Farah, 2007). The parents' qualifications were converted to a scale from 0 to 5 ( $0=$ no formal education and $5=$ university degree). In families with an absent mother or father, the score of the present parent was considered as the family score. Parental occupational status was coded following the International Standard Classification of Occupations (ISCO, 2008). Unit groups (one-digit) only codes were inverted and retained for analyses of occupational status, with scores ranking from 1 (unemployed) to 10 (administrative and commercial managers) in order of ascending occupational prestige. A family mean occupational score was derived from both parents' ISCO-08 scores, but if one parent was absent, the score of the present parent was considered to be the family score. The family income wage distribution was obtained, considering the wage distribution in Macau in 2014 (DSEC, 2014), and split into six levels that included equal proportions of the workforce. To calculate the income-to-need measure, we transformed the income range values in average values and divided these between the consumer-units of each family. ${ }^{1}$

A principal components analysis was carried out on the entire dataset using the three main SES variables (i.e., education level, occupation level, and income-to-need measure) free of missing data. For a three-component solution, only the first component was associated to a self-value higher than 1. All three variables loaded evenly on this component, which explained $60 \%$ of total variance. Factor 1 was therefore the only one retained, and applying the regression method, coordinates of each individual on the first component of the resulting principal component analysis was included as a global index of socioeconomic status. The global index of SES was used as moderator and a continuous variable to test the hypothesis of a relation between authoritative parenting style and informational processing style, and the researchers conducted a moderated path analysis recommended by Hayes (2013). In this regard, the SES was separated into five levels (10th, 25 th, 50th, 75th, and 90th) to see whether the relation between authoritative parenting and informational identity processing would differ. In addition, we also explored how authoritative parenting would influence the relation between SES and informational style by separating the authoritative parenting style into five levels to examine possible differences in the relation between SES and informational processing. 


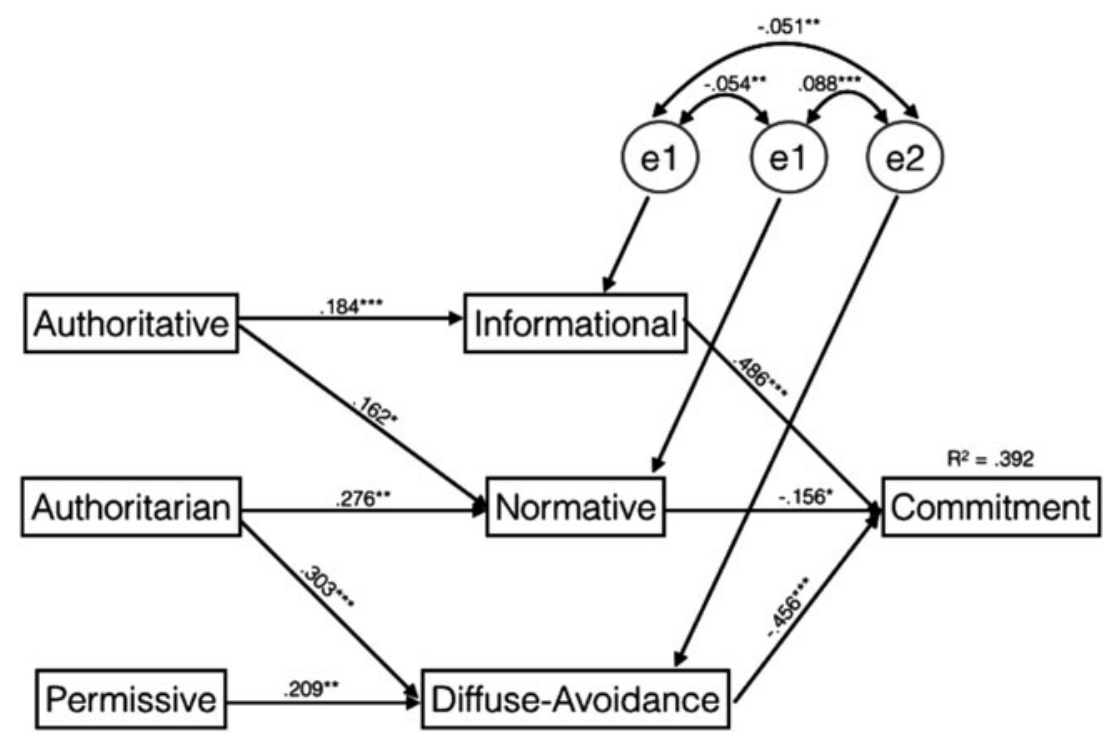

\section{Figure 2}

Model 2: The revised model for Macau youth with the path authoritative to diffuse-avoidant from original Berzonsky (2004) model removed. Note: ${ }^{*} p<.05,{ }^{* *} p<.01,{ }^{* * *} p<.001$

\section{Results}

The results of this research are presented in two main sections. First, we present the data obtained contrasting Berzonsky's model (2004) in our sample, showing the fit indices of the revised model for Macau youth. Subsequently, we tested a moderated path model using SES as the moderator to examine the relationship between the perceived authoritative parenting style and informational identity processing if the family had a high SES.

\section{Perceived Parenting Styles and Identity Processing Among Macau Youth}

A path analysis was conducted to test the fit of Berzonsky's model (2004) regarding the relationship between parental authority and identity processing styles and commitment for Macau youth. The path model suggested by Berzonsky (2004; see Figure 1) was tested with a robust maximumlikelihood analysis (Satorra \& Bentler, 1994) using R script (package 'lavaan'; Rosseel, 2012). The initial test showed a poor-fitting model with most indices not meeting the standards $\left(\chi^{2}=32.936, d f=8, p<.001 ;\right.$ RMSEA $=.122$; SRMR $=.071 ; \mathrm{CFI}=.836)$. Within Berzonsky's (2004) model the three parental styles were correlated, but only the Informational and the Diffuse-Avoidant scales were correlated. In our data, the three parental style scales were also correlated (all ps <.01). As in the original model, these correlations are not shown in the figures. However, we found that the three identity processing style scales from the ISI-4 questionnaire should be covaried with each other. We also found that in our data there was no significant correlation between the authoritative parenting and diffuse-avoidant processing $(\beta=.005, p=.946)$. This implied a somewhat adapted structure for identity styles in
Macau participants. Thus, dropping the authoritative to diffuse-avoidant path, and adding the covariance between the three error terms of the identity styles gave a good model fit $\left(\chi^{2}=9.205, d f=7, p=.238\right.$; RMSEA $=.039$; SRMR $=.026$; CFI $=.985)$ with a sample size above 200 $(N=209)$. The revised model for Macau youth with standardised path coefficients maintained the basic structure of the original model proposed by Berzonsky (2004) and was used for further analysis (see Figure 2).

Model 1 (Figure 1) and Model 2 (Figure 2) were compared using the model fit indices and the chi-square statistic (see Table 1). We also used the chi-square difference test (Bollen, 1989) to calculate the difference between the Berzonsky's model (2004) and the revised model $\left(\Delta \chi^{2}=\right.$ 24.213, $d f=1, p<.001)$. The results showed that the revised model (Model 2) fitted the data better. The path coefficients for the two models included the path descriptions, unstandardised coefficients, the standard errors, and the standardised coefficients are reported in Table 3.

The results of the comparative analysis - that is, comparing Berzonsky's (2004) model (Figure 1) and our adapted model (Figure 2) — showed that the adapted model could be applied to test the relationship between parental authority, identity processing styles, and commitment among college students in Macau. As predicted, we found a positive relation between perceived authoritative parenting and the informational style $(\beta=.184, p<$ $.001)$, and a positive relation between authoritative parenting and the normative style $(\beta=.162, p=.014)$ but, as noted before, no relation between perceived authoritative parenting and the diffuse-avoidant processing style. Regarding the perceived authoritarian parenting style, we obtained the hypothesised positive relation with the normative processing style $(\beta=.276, p<.001)$ and with 
Table 1

Summary of Model Fit Statistics for Model 1 (Berzonsky, 2004) and Model 2 (Revised)

\begin{tabular}{|c|c|c|c|c|}
\hline \multirow[b]{2}{*}{ Model fit index } & \multicolumn{2}{|c|}{ Model 1} & \multicolumn{2}{|c|}{ Model 2} \\
\hline & $\mathrm{ML}$ & Robust & $\mathrm{ML}$ & Robust \\
\hline$x^{2}$ test & 37.002 & 32.936 & 10.372 & 9.205 \\
\hline$d f$ & 8 & 8 & 7 & 7 \\
\hline$p$ & $<.001$ & $<.001$ & .168 & .238 \\
\hline $\mathrm{CFI}$ & .839 & .836 & .981 & .985 \\
\hline TLI & .637 & .630 & .952 & .963 \\
\hline RMSEA & .132 & .122 & .048 & .039 \\
\hline $90 \% \mathrm{Cl}$ & {$[.091, .176]$} & {$[.083, .164]$} & {$[.000, .105]$} & {$[.000, .096]$} \\
\hline AIC & 2249.0 & 2249.0 & 2224.4 & 2224.4 \\
\hline $\mathrm{BIC}$ & 2309.2 & 2309.2 & 2227.7 & 2227.7 \\
\hline SRMR & .071 & .071 & .026 & .026 \\
\hline
\end{tabular}

Note: $\mathrm{ML}=$ Maximum likelihood estimates; $\chi^{2}$ test $=$ chi-square test; $\mathrm{CFI}=$ comparative fit index; $\mathrm{TLI}=$ Tucker-Lewis index; RMSEA $=$ root mean square error of approximation; $\mathrm{AIC}=$ Akaike information criterion; $\mathrm{BIC}=$ Bayesian information criterion; SRMR $=$ standardised root mean square residual.

diffuse-avoidant processing style $(\beta=.303, p<.001)$. The path between the perceived permissive parenting style and the diffuse-avoidant processing style also showed a positive relation $(\beta=.209, p=.006)$. Furthermore, the results showed the hypothesised positive relation between informational processing style and identity commitment ( $\beta=$ $.486, p<.001)$, and the negative relation between diffuseavoidant style and commitment $(\beta=-.456, p<.001)$. However, different from the original model by Berzonsky (2004), the results from our revised model for Macau youth showed a negative relation between normative processing style and identity commitment $(\beta=-.156, p=$ $.027)$. Covariances among identity processing styles' residuals were negative between informational and the normative and diffuse-avoidant processing styles $(\beta=-.054, p$ $<.001 ; \beta=-.051, p<.001$, respectively), and positive between normative and diffuse-avoidant processing styles ( $\beta=.088, p<.001)$. The possible reasons for the differences in covariances between Berzonsky's model and the revised model are discussed below.

\section{Moderated Effect of SES on the Relation Between Authoritative Parenting and Informational Style}

We also examined the moderating effect of the family SES on the relation between authoritative parenting and the informational processing style. Using the revised model for Macau youth (Figure 2), we tested Model 3 with path $b_{2}$ (SES index -> Informational) and $\mathrm{b}_{3}$ (Authoritative $*$ SES -> Informational) constrained to be 0 , Model 4 with path $\mathrm{b}_{3}$ (Authoritative $*$ SES - $>$ Informational) constrained to be 0 , and Model 5 with no path constrained. The difference between Model 3 and Model 4 was not significant $\left(\Delta \chi^{2}=\right.$ $.533, d f=1, p=.47)$, but when compared with Model 5, the differences were significant (Model 3 vs. Model 5: $\Delta \chi^{2}$ $=6.55, d f=1, p=.04$; Model 4 vs. Model 5: $\Delta \chi^{2}=5.55$, $d f=1, p=.02)$. The results in Table 2 suggested that the moderated path model performed very well $\left(\chi^{2}=16.098\right.$, $d f=13, p>.05$; RMSEA $=.034$; SRMR $=.033$; CFI $=.981$ ), supporting the moderation effect of SES in the relation between parenting and identity processing style.
The path coefficients of the moderated path model are shown in Table 4.

Figure 3 illustrates the moderated path model with the standardised path coefficient $\left(\Theta_{\mathrm{X}-\mathrm{Y}}=.178+.121 \mathrm{Mod}\right)$ and explains the relationship between authoritative parenting style (X) and the informational processing style (Y) as a function of SES index (Hayes, 2013). The SES index ranged from -2.25 to $2.28\left(\Theta_{\mathrm{X}->\mathrm{Y}}-.09\right.$ to .45$)$. We selected the 10th, 25th, 50th, 75th, and 90th percentiles as the Mod values, and which resulted in $\operatorname{Mod}=-1.204, \Theta_{\mathrm{X}->\mathrm{Y}}=.03$, $\operatorname{Mod}=-.730, \Theta_{\mathrm{X}->\mathrm{Y}}=.09, \operatorname{Mod}=-.100, \Theta_{\mathrm{X}->\mathrm{Y}}=.17$, $\operatorname{Mod}=.640, \Theta_{\mathrm{X}->\mathrm{Y}}=.26, \mathrm{Mod}=1.490, \Theta_{\mathrm{X}->\mathrm{Y}}=.36$ respectively for the five selected percentiles. The results indicated that the higher the SES index, the stronger the relationship between the authoritative parenting style and the informational processing style.

To explain the relationship between the SES index and the informational processing style as a function of authoritative parenting, we used $\Theta_{\text {Mod- }>\mathrm{Y}}=-.405+.121 \mathrm{X}$. The perceived authoritative parenting ranged from 1.55 to 4.85 and the $\Theta_{\text {Mod- }>Y}$ ranged from - .22 to .18. We again selected the 10th, 25th, 50th, 75th, and 90th percentiles for the authoritative parenting style value $(\mathrm{X})$, which resulted in $\mathrm{X}=2.55, \Theta_{\text {Mod- }>\mathrm{Y}}=-.10, \mathrm{X}=2.95, \Theta_{\text {Mod- }>\mathrm{Y}}=-.05, \mathrm{X}$ $=3.40, \Theta_{\text {Mod }->\mathrm{Y}}=.01, \mathrm{X}=3.90, \Theta_{\text {Mod }->\mathrm{Y}}=.07$, and $\mathrm{X}=$ 4.25, $\Theta_{\text {Mod->Y }}=.11$. Only a higher score for authoritative parenting $\mathrm{X}$ above 3.35 showed a strong positive relationship between SES and the informational processing style. When the score for authoritative parenting was $\mathrm{X}$ below 3.35 , there was a stronger negative relationship between the SES and the informational processing style.

\section{Discussion}

In this study, we examined the model proposed by Berzonsky (2004) to investigate the relationship between parenting styles and the socio-cognitive processing styles and identity commitment among college students in Macau. Limited information existed about whether or not Berzonsky's model would fit a population of predominantly 
Table 2

Path Coefficients of Model 1 (Berzonsky, 2004) and Model 2 (Revised)

\begin{tabular}{|c|c|c|c|c|}
\hline Parameter & Unstandardised & SE & Standardised & $p$ value \\
\hline & Model 1 & & & \\
\hline \multicolumn{5}{|l|}{ Regressions } \\
\hline Authoritative - $>$ Informational & .182 & .050 & .261 & .000 \\
\hline Authoritative -> Normative & .161 & .070 & .198 & .021 \\
\hline Authoritative -> Diffuse & .005 & .077 & .006 & .946 \\
\hline Authoritarian -> Normative & .265 & .078 & .290 & .001 \\
\hline Authoritarian -> Diffuse & .314 & .078 & .331 & .000 \\
\hline Permissive -> Diffuse & .233 & .098 & .205 & .017 \\
\hline Informational -> Commitment & .486 & .072 & .352 & .000 \\
\hline Normative -> Commitment & -.156 & .063 & -.132 & .013 \\
\hline Diffuse -> Commitment & -.456 & .067 & -.401 & .000 \\
\hline \multicolumn{5}{|l|}{ Covariance } \\
\hline Informational $<->$ Diffuse & -.052 & .017 & -.209 & .002 \\
\hline \multicolumn{5}{|l|}{ Intercepts } \\
\hline Informational & 3.438 & .173 & 7.312 & .000 \\
\hline Normative & 1.777 & .407 & 3.230 & .000 \\
\hline Diffuse & 1.472 & .422 & 2.576 & .000 \\
\hline \multirow[t]{2}{*}{ Commitment } & 3.303 & .437 & 5.084 & .000 \\
\hline & Model 2 & & & \\
\hline \multicolumn{5}{|l|}{ Regressions } \\
\hline Authoritative -> Informational & .184 & .049 & .263 & .000 \\
\hline Authoritative -> Normative & .162 & .066 & .198 & .014 \\
\hline Authoritarian -> Normative & .276 & .073 & .302 & .000 \\
\hline Authoritarian -> Diffuse & .303 & .074 & .320 & .000 \\
\hline Permissive -> Diffuse & .209 & .076 & .184 & .006 \\
\hline Informational -> Commitment & .486 & .074 & .334 & .000 \\
\hline Normative -> Commitment & -.156 & .071 & -.129 & .027 \\
\hline Diffuse -> Commitment & -.456 & .073 & -.390 & .000 \\
\hline \multicolumn{5}{|l|}{ Covariance } \\
\hline Informational $<->$ Diffuse & -.051 & .071 & -.208 & .002 \\
\hline Informational $<->$ Normative & -.054 & .019 & -.225 & .005 \\
\hline Normative $<->$ Diffuse & .088 & .021 & .305 & .000 \\
\hline \multicolumn{5}{|l|}{ Intercepts } \\
\hline Informational & 3.431 & .171 & 7.292 & .000 \\
\hline Normative & 1.742 & .376 & 3.158 & .000 \\
\hline Diffuse & 1.601 & .388 & 2.814 & .000 \\
\hline Commitment & 3.303 & .442 & 4.964 & .000 \\
\hline
\end{tabular}

Note: $\mathrm{SE}=$ standard error.

Table 3

Results From Various Regression Models in Moderated Path Analysis

\begin{tabular}{|c|c|c|c|c|c|c|}
\hline \multirow[t]{2}{*}{ Model } & \multicolumn{2}{|c|}{ Model 3} & \multicolumn{2}{|c|}{ Model 4} & \multicolumn{2}{|c|}{ Model 5} \\
\hline & Coeff. & $p$ value & Coeff. & $p$ value & Coeff. & $p$ value \\
\hline Authoritative $(X) b_{1}$ & .184 & .000 & .170 & .001 & .178 & .000 \\
\hline SES index $(M) b_{2}$ & & & .024 & .456 & -.405 & .011 \\
\hline Informational $(Y) b_{3}$ & & & & & .121 & .007 \\
\hline Model fit index & ML & Robust & $\mathrm{ML}$ & Robust & ML & Robust \\
\hline$\overline{\chi^{2} \text { test }}$ & 23.943 & 22.921 & 23.405 & 22.353 & 16.643 & 16.098 \\
\hline$d f$ & 15 & 15 & 14 & 14 & 13 & 13 \\
\hline$p$ & .066 & .086 & .054 & .072 & .216 & .244 \\
\hline CFI & .952 & .950 & .949 & .948 & .980 & .981 \\
\hline TLI & .916 & .914 & .906 & .903 & .961 & .961 \\
\hline RMSEA & .053 & .050 & .057 & .053 & .037 & .034 \\
\hline $90 \% \mathrm{Cl}$ & {$[.00, .09]$} & {$[.00, .09]$} & {$[.00, .10]$} & {$[.00, .09]$} & {$[.00, .08]$} & {$[.00, .08]$} \\
\hline AIC & 3191.7 & 3191.7 & 3193.1 & 3193.1 & 3188.4 & 3188.4 \\
\hline $\mathrm{BIC}$ & 3255.2 & 3255.2 & 3260.0 & 3260.0 & 3258.6 & 3258.6 \\
\hline SRMR & .037 & .037 & .033 & .033 & .033 & .033 \\
\hline
\end{tabular}

Note: $\mathrm{ML}=$ Maximum likelihood robust estimates; $\chi^{2}$ test $=$ chi-square test; $\mathrm{CFI}=$ comparative fit index; TLI $=$ Tucker-Lewis index; RMSEA $=$ root mean square error of approximation; $\mathrm{AIC}=$ Akaike information criterion; $\mathrm{BIC}=$ Bayesian information criterion; SRMR $=$ standardised root mean square residual. 
Table 4

Path Coefficients of Moderated Path Model

\begin{tabular}{|c|c|c|c|c|}
\hline Parameter & Unstandardised & SE & Standardised & $p$ value \\
\hline \multicolumn{5}{|l|}{ Regressions } \\
\hline Authoritative -> Informational & .178 & .046 & .257 & .000 \\
\hline SES -> Informational & -.405 & .159 & -.874 & .011 \\
\hline Authoritative * SES -> Informational & .121 & .045 & .928 & .007 \\
\hline Authoritative -> Normative & .162 & .066 & .199 & .014 \\
\hline Authoritarian -> Normative & .278 & .074 & .303 & .000 \\
\hline Authoritarian-> Diffuse & .305 & .075 & .323 & .000 \\
\hline Permissive -> Diffuse & .212 & .076 & .186 & .005 \\
\hline Informational -> Commitment & .486 & .074 & .343 & .000 \\
\hline Normative -> Commitment & -.156 & .070 & -.130 & .026 \\
\hline Diffuse -> Commitment & -.456 & .073 & -.391 & .000 \\
\hline \multicolumn{5}{|l|}{ Covariance } \\
\hline Informational <-> Diffuse & -.048 & .018 & -.203 & .008 \\
\hline Informational $<->$ Normative & -.049 & .016 & -.203 & .003 \\
\hline Normative $<->$ Diffuse & .088 & .021 & .305 & .000 \\
\hline \multicolumn{5}{|l|}{ Intercepts } \\
\hline Informational & 1.738 & .380 & 3.148 & .000 \\
\hline Normative & 1.583 & .389 & 2.780 & .000 \\
\hline Diffuse & 3.417 & .158 & 7.300 & .000 \\
\hline Commitment & 3.303 & .436 & 4.974 & .000 \\
\hline
\end{tabular}

Note: SE = standard error, SES = socioeconomic status.

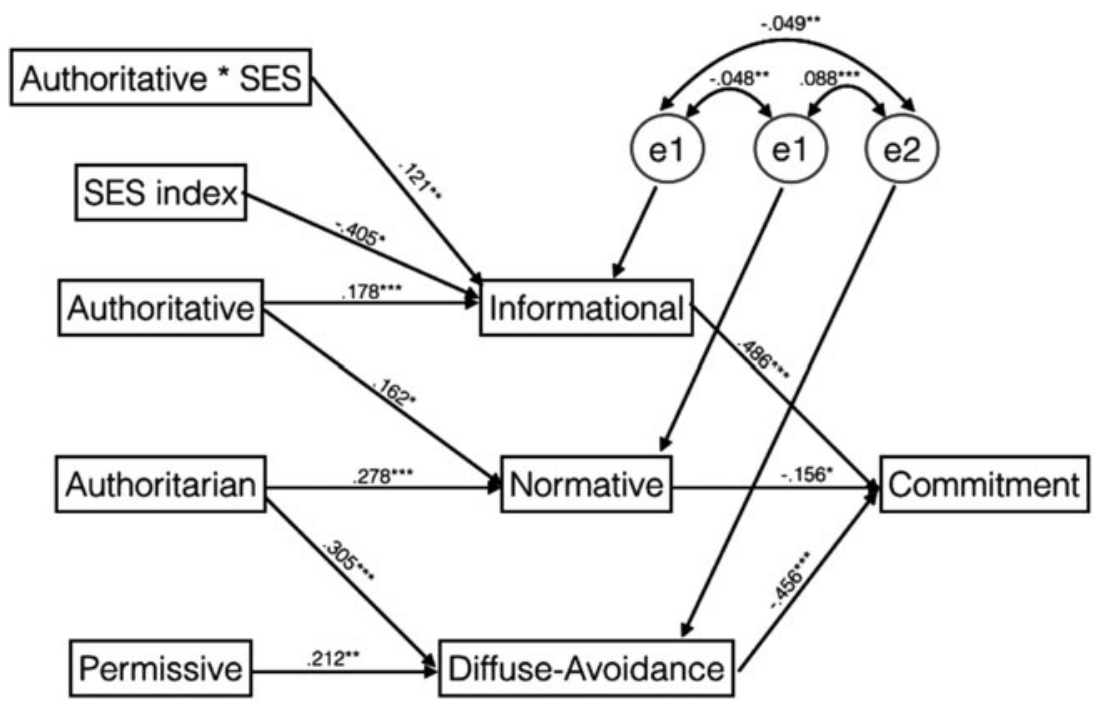

Figure 3

Moderated path model including the relationship between authoritative parenting, SES and the informational processing style.

Note: $* p<.05, * * p<.01,{ }^{* * *} p<.001$.

Chinese heritage. Thus, the first analysis was done to find a model fit between Berzonsky's model and the sample included in this study. The results showed that the path from authoritative parenting to diffuse-avoidant styles was not significant, and the model was adapted using a correction factor (Satorra \& Bentler, 1994) and model fit indices (Beaujean, 2014; West, Taylor, \& Wu, 2012) to find a fit. To better fit the data, we proposed that the revised model should drop the path from authoritative parenting to diffuse-avoidant style and not only correlate the error terms for informational and diffuse-avoidant scales, but also include error terms for the correlation between the informational and normative style, and the correlation between the normative and diffuse-avoidance style. Comparing Berzonsky's model with the revised model using the chi-square test showed that the revised model fit the data better. Thus, we could maintain the basic structure of the model proposed by Berzonsky (2004) to examine the relationship between parental authority and identity processing and commitment. The revised model also posed a better fit for the cultural context in Macau and the ways local youth perceived their identity processing somewhat differently from young people in Western countries.

The results using the better fit model confirmed the hypotheses that perceived authoritative parenting style was positively correlated with the informational processing 
style and the normative processing style, but not with the diffuse-avoidant processing style. As predicted, the authoritarian parenting style positively correlated with the normative processing style and with diffuse-avoidant processing, and permissive parenting was positively correlated with the diffuse-avoidant processing style. Thus, we concluded, as did Berzonsky (2004) and others (e.g., Berzonsky, Branje, \& Meeus, 2007), that youth from authoritative families were more likely to use the information processing style, perceiving their parents as more responsive to their needs and forthcoming with information necessary for identity commitment. The youth from authoritarian families adopted a normative processing style, perceiving their parents as less responsiveness and possibly more demanding of conformity to existing norms in society. Individuals perceiving their parents as permissive were more inclined towards a diffuse-avoidant social cognitive identity processing style, given that their parents were purportedly less demanding although moderately communicative. Furthermore, the strong positive correlation between authoritative and permissive parenting styles needs further exploration and questioning of the parenting typology commonly used in Western countries. Chinese youth apparently do not perceive their parents as 'permissive' when the parents pose fewer demands and controls on their socio-cognitive explorations, focusing rather on the parents' responsiveness to their needs. On the other hand, the demandingness embedded within the authoritative parenting style is perceived as benevolent control and supportive of socio-cognitive explorations (Chen, Dong, \& Zhou, 1997; Lee, Pratto, \& Li, 2007).

The results from the revised structural model corroborated with findings in previous Western studies for identity commitment (Adams, Berzonsky, \& Keating, 2006; Berzonsky et al., 2007). Macau youths adopting the informational style showed a positive relation to identity commitment. However, in contrast to Western studies, our results showed a negative relationship between the normative socio-cognitive processing style and identity commitment, which suggested that individuals with this processing style did not express a clear identity commitment. This also contradicted previous studies claiming that individuals who employed the normative processing style were likely to form a foreclosed identity commitment earlier in adolescence (Berzonsky, 2004; Berzonsky et al., 1999; Berzonsky et al., 2007). One explanation is that, in the local context, normative individuals are overprotected and tend to exhibit a lack of independence and responsibility for their own decisions (Lee et al., 2007; Papini, Micka, \& Barnett, 1989; Perosa, Perosa, \& Tam, 1996). As a result they might delay forming identity commitment even when entering university.

Apart from investigating the relationship between parental authority and identity processing styles, we also examined the moderating effect of socioeconomic status on parenting styles and the informational processing style of Macau youth. Macau is widely considered a wealthy city with low unemployment ( $>2 \%$ ), monthly earnings of $14,000 \mathrm{MOP}$ per employed person, and main employment in the services industry requiring secondary education only (DSEC, 2014). We predicted that family SES - that is, the composite number for family education level, occupation levels, and income-to-need measure would differentially impact the identity commitment of youth perceiving their parents as authoritative. The results from the moderated path model (Figure 3) supported the hypothesis that if students came from a higher SES family (above the 50th percentile), they most likely had parents adopting an authoritative parenting style and used the informational processing $\left(\Theta_{\text {Mod- }>Y}=.01, \Theta_{\text {Mod- }>\mathrm{Y}}=\right.$ $.07, \Theta_{\text {Mod- }>Y}=.11$ ). In lower SES families (below 50th percentile) the parents could also adopt the authoritative parenting style but the students in this group would not necessarily use the informational socio-cognitive processing $\left(\Theta_{\text {Mod- }>\mathrm{Y}}=-.10, \Theta_{\text {Mod- }>\mathrm{Y}}=-.05\right)$, perhaps because resources were limited in lower SES families. Thus, we concluded that a higher SES family where the parents adopted an authoritative style of parenting would support the young person's need for information and resources in identity processing and commitment.

However, if the parents were less authoritative, the high SES did not influence the young person to use informational socio-cognitive processes for identity commitment. Lower SES likely limits the availability of social and economic resources for identity processing, leading to a strong negative relationship between SES and the informational processing style. The family SES not only measured economic factors (income-to-need) but also the educational level and occupational prestige of parents. Thus, although parents might be perceived as authoritative and had sufficient income-to-needs, it was likely that they did not have high educational levels or occupations demanding higher education, and therefore the parents were among the lower percentiles for SES. Despite its current wealth, the majority of jobs in Macau are in the hospitality industry and gaming, which do not have high rankings on the ISCO-08 (ISCO, 2008). Thus, authoritative parents with lower SES were responsive to the young person's needs and allowed a certain degree of independence for decision-making, but the information necessary for identity processing and guidance for problem solving were less forthcoming.

As expected, there was a strong relationship between perceived authoritative parenting and the informational processing style when moderated by the SES effect, particularly if an individual perceived her or his parents had a higher score on both SES and authoritativeness. Individuals who were from higher socioeconomic groups perceived their parents as authoritative, and these youth were more likely to adopt the informational style in their identity commitment process. This could be explained by the notion that parents coming from the higher SES group were most likely to have higher education levels and greater wealth, and engaged more frequently in supportive childrearing practices while respecting their child's search for 
autonomy and freedom (Gauvain \& Huard, 1999; Zhou et al., 2004). Although they are demanding, authoritative parents are highly responsive, and in this regard, Macau parents are presumably similar to their counterparts in the West. The higher the SES, the more likely that parental authority was perceived as being responsive to the youth's needs and the more forthcoming the resources were for adopting an informational processing style and identity commitment. The young people were also likely to perceive their parents' style as deferential and benevolent (Lee et al., 2007), and as supportive of their socio-cognitive processing during the identity development phase. On the contrary, and as expected, when high SES families were perceived as having low authoritative parenting style, the influence of the socio-economic status disappeared. Participants who perceived their parents as medium or low in authoritative parenting were less likely to adopt the informational identity style.

However, we could not generalise the results of this study to all youth in Macau, given that we only recruited participants who were studying at a local tertiary institution in Macau. Without a randomised sample across all sectors of the emerging adult population in Macau, we could not generalise the findings to those who did not, could not, or chose not to pursue further education after school. The results are therefore limited to university students who were more likely to perceive their parents as authoritative and came from higher SES families. Although the sample size was large enough to have good statistical power to test Berzonsky's (2004) model, we were unable to further explore the SES moderator effect on other paths.

Furthermore, an inherent feature of the selfadministered and retrospective questionnaires was that the information obtained was not free of bias due to social desirability, memory and personality, particularly with regard to retrieving autobiographical information about parenting styles. We also have to take into consideration that although we used bilingual versions of the questionnaires translated by experts in the field, those translations did not account for a complete adaptation of the instruments to the cultural and linguistic characteristics of the Chinese population. These features further limited the generalisability of the findings to the larger population of Chinese youth in Macau. The sample was also skewed with more females than males, and we did not test for gender differences being limited by the length of this report.

Further studies are needed to test the revised model with new data and investigate the SES moderation effect. This would allow researchers to support the revised model to examine identity styles within a Chinese context and to further explicate the differences in perceived identity processing between Asian and Western youth. Further research on this topic should also involve a gender-balanced and randomised sample of all Chinese youth in Macau. A larger sample size will allow for in-depth validation of the
ISI-4 and the model for identity processing styles tested with a revised typology for perceived parenting styles that is more relevant to Chinese parental engagement with their children. Bigger sample sizes of different SES groups should be included to increase the power of the findings of this study. Ultimately, we encourage future researchers to contrast the adaptability of our revision of Berzonsky's model with other Asian populations, taking into account not only SES variability, but also broader age ranges and longitudinal samples, to allow insights about the evolution of the identity processing styles during late adolescent and emerging adulthood development.

\section{Acknowledgments}

This research received no specific grant from any funding agency, commercial or not-for-profit sectors. One of the authors was supported by an Erasmus Mundus MULTI grant (Action 2 Strand 2, Multilingualism and Multiculturalism) from the European Commission for an exchange program at the University of Macau. We also want to acknowledge the collaboration of the staff of the Department of Psychology of the University of Macau and the students of the Faculty of Social Sciences who participated in this study.

\section{Endnote}

1 Following OECD recommendations, consumer units were calculated adding (number of adults $* 0.75)+$ (number of children under 18 years $* 0.5$ ) of each family set.

\section{References}

Adams, G.R., Berzonsky, M.D., \& Keating, L. (2006). Psychosocial resources in first-year university students: The role of identity processes and social relationships. Journal of Youth and Adolescence, 35, 78-88. doi:10.1007/s10964-005-9019-0

American Psychological Association Task Force on Socioeconomic Status. (2007). Report of the APA Task Force on Socioeconomic Status. Washington, DC: Author.

Barcinski, M., \& Kalia, V. (2005). Extending the boundaries of the dialogical self: Speaking from within the feminist perspective. Culture \& Psychology, 11, 101-109 doi:10.1177/1354067×05050752

Barnes, S.P., Brown, K.M., McDermott, R.J., Bryant, C.A., \& Komrey, J. (2012). Perceived parenting style and the eating practices of college freshmen. American Journal of Health Education, 43, 8-17. doi:10.1080/19325037.2012.10599213

Baumrind, D. (1991). Parenting styles and adolescent development. In J. Brooks-Gunn, R. Lerner, \& A.C. Peterson (Eds.), The Encyclopedia of Adolescence (pp. 746-758). New York: Garland.

Beaujean, A. (2014). Latent variable modelling using R: A stepby-step guide. New York: Routledge.

Bentler, P.M., \& Chou, C.P. (1987). Practical issues in structural modeling. Sociological Methods Research, 16, 78-117. doi:10.1177/0049124187016001004 
Bednar, D.E., \& Fisher, T.D. (2003). Peer referencing in adolescent decision making as a function of perceived parenting style. Adolescence, 38, 607-621.

Berzonsky, M.D. (1989). Identity style: Conceptualization and measurement. Journal of Adolescent Research, 4, 268-282. doi:10.1177/074355488943002

Berzonsky, M.D. (1990). Self-construction over the life-span: A process perspective on identity formation. In G.J. Neimeyer \& R.A. Neimeyer (Eds.), Advances in personal construct psychology (vol. 1, pp. 155-186). Greenwich, CT: JAI.

Berzonsky, M.D. (2004). Identity styles, parental authority, and identity commitment. Journal of Youth and Adolescence, 33, 213-220. doi:10.1023/B:JOYO.0000025320.89778.29

Berzonsky, M.D., Branje, S.J.T., \& Meeus, W. (2007). Identityprocessing style, psychosocial resources, and adolescents' perceptions of parent adolescent relations. Journal of Early Adolescence, 27, 324-345. doi:10.1177/0272431607302006

Berzonsky, M.D., \& Ferrari, J.R. (1996). Identity orientation and decisional strategies. Personality and Individual Differences, 20, 597-606. doi:10.1016/0191-8869(96)00001-3

Berzonsky, M.D., \& Kuk, L. (2000). Identity status, identity processing style, and the transition to university. Journal of Adolescent Research, 15, 81-98. doi:10.1177/0743558400151005

Berzonsky, M.D., \& Neimeyer, G.J. (1994). Ego identity status and identity processing orientation: The moderating role of commitment. Journal or Research in Personality, 28, 425-435. doi:10.1006/jrpe.1994.1030

Berzonsky, M.D., Nurmi, J.E., Kinney, A., \& Tammi, K. (1999). Identity processing styles and cognitive attributional strategies: Similarities and difference across different contexts. European Journal of Personality, 13, 105-120. doi:10.1002/(SICI)1099-0984(199903/04)13:2 < 105::AIDPER325>3.0.CO;2-H

Bollen, K.A. (1989). A new incremental fit index for general structural equation models. Sociological Methods \& Research, 7, 303-316. doi:10.1177/0049124189017003004

Bond, M.H. (1991). Beyond the Chinese face. Hong Kong: Oxford University Press.

Bradley, R.H., \& Corwyn, R.F. (2002). Socioeconomic status and child development. Annual Review of Psychology, 53, 371399. doi:10.1146/annurev.psych.53.100901.135233

Bronfenbrenner, U., \& Ceci, S.J. (1994). Nature-nurture reconceptualized in developmental perspective: A bioecological model. Psychological Review, 101, 568-586. doi:10.1037/0033-295X.101.4.568

Buri, J.R. (1991). Parental Authority Questionnaire. Journal of Personality Assessment, 57, 110-119. doi:10.1207/s15327752jpa5701_13

Chang, L., McBride-Chang, C., Stewart, S.M., \& Au, E. (2003). Life satisfaction, self-concept, and family relations in Chinese adolescents and children. International Journal of Behavioral Development, 27, 182-189. doi:10.1080/01650250244000182

Chang, L., Schwartz, D., Dodge, K.A., \& McBride-Chang, C. (2003). Harsh Parenting in Relation to Child Emotion Regulation and Aggression. Journal of Family Psychology, 17, 598-606. doi:10.1037/0893-3200.17.4.598
Chen, R.K., Dong, Q., \& Zhou, H. (1997). Authoritative and authoritarian parenting practices and social and school performance in Chinese children. International Journal of Behavioral Development, 21, 855-873. doi:10.1080/016502597384703

Darling, N., \& Steinberg, L. (1993). Parenting style as context: An integrative model. Psychological Bulletin, 113, 487-496. doi:10.1037/0033-2909.113.3.487

DSEC. (2014). Government of Macau Special Administrative Region Statistics and Census Service. Retrieved April 2015 from http://www.dsec.gov.mo/default.aspx?lang=en-US

Erikson, E.H. (1986), Identity: Youth and crisis. New York: Norton.

Farah, M.J., Shera, D.M., Savage, J.H., Betancourt, L., Giannetta, J.M., Brodsky, N.L., ... Hurt, H. (2006). Childhood poverty: Specific associations with neurocognitive development. Brain Research, 1110, 166-174. doi:10.1016/j.brainres.2006.06.072

Gauvain, M., \& Huard, R.D. (1999). Family interaction, parenting style, and the development of planning: A longitudinal analysis using archival data. Journal of Family Psychology, 13, 75-92. doi:10.1037/0893-3200.13.1.75

Georgas, J. (2003). Family: Variations and changes across cultures. In W.J. Lonner, D.L. Dinnel, S.A. Hayes, \& D.N. Sattler (Eds.), Online Readings in Psychology and Culture (Unit 13, Chapter 3). Bellingham, WA: Center for Cross-Cultural Research, Western Washington University.

Fernald, A., Marchman, V.A., \& Weisleder, A. (2013). SES differences in language processing skill and vocabulary are evident at 18 months. Developmental Science, 16, 234-248.

Hackman, D.A., \& Farah, M.J. (2009). Socioeconomic status and the developing brain. Trends in Cognitive Sciences, 13, 65-73. doi:10.1016/j.tics.2008.11.003

Hanson, J.L., Hair, N., Shen, D.G., Shi, F., Gilmore, J. H., Wolfe, B.L., \& Pollak, S.D. (2013). Family poverty affects the rate of human infant brain growth. PloS One, 8, e80954. doi:10.1371/journal.pone.0080954

Hayes, A.F. (2013). Introduction to mediation, moderation, and conditional process analysis: A regression-based approach. Guilford Press.

Hoff, E., Laursen, B., \& Tardif, T. (2002). Socioeconomic status and parenting. In M.H. Bornstein (Ed.), Handbook of parenting (Vol. 2, pp. 231-252). Mahwah, NJ: Erlbaum.

Hollingshead, A.A. (1975). Four Factor Index of Social Status. Unpublished manuscript, Yale University, New Haven, CT.

ISCO. (2008). International Standard Classification of Occupations 2008 (ISCO-08). New York: United Nations, Department of Economic and Social Affairs.

Jackson, L.M., Pratt, M.W., \& Hunsberger, B. (2005). Optimism as a mediator of the relation between perceived parental authoritativeness and adjustment among adolescents: Finding the sunny side of the street. Social Development, 14, 273-304. doi:10.1111/j.1467-9507.2005.00302.x

Kagitcibasi, C. (2002). A model of family change in cultural context. In W.J. Lonner, D.L. Dinnel, S.A. Hayes, \& 
D.N. Sattler (Eds.), Online Readings in Psychology and Culture (Unit 13, Chapter 1). Bellingham, WA: Center for CrossCultural Research, Western Washington University.

Kishiyama, M.M., Boyce, W.T., Jimenez, A.M., Perry, L.M., \& Knight, R.T. (2009). Socioeconomic disparities affect prefrontal function in children. Journal of Cognitive Neuroscience, 21, 1106-1115. doi:10.1162/jocn.2009.21101

Kline, R.B. (2005). Principles and practice of structural equation modeling (2nd ed.). New York: Guilford Press.

Lawford, H., Pratt, M.W., \& Hunsberger, B. (2005). Adolescent Generativity: A longitudinal study of two possible contexts for learning concern for future generations. Journal of Research on Adolescence, 15, 261-273. doi:10.1111/j.1532-7795.2005.00096.x

Lee, I.C., Pratto, F., \& Li, M.C. (2007). Social relationships and sexism in the United States and Taiwan. Journal of Cross-Cultural Psychology, 38, 595-612. doi:10.1177/0022022107305241

Lerner, J.V., \& Castellino, D.R. (2000). Parent-child relationship: Childhood. In A.E. Kazdin (Ed.), Encyclopedia of psychology (vol. 6). Washington, DC: American Psychological Association.

Letourneau, N. L., Duffett-Leger, L., Levac, L., Watson, B., \& Young-Morris, C. (2013). Socioeconomic Status and Child Development: A Meta-Analysis. Journal of Emotional and Behavioral Disorders, 21, 211-224. doi:10.1177/1063426611421007

Leung, K., Lau, S., \& Lam, W.L. (1998). Parenting styles and academic achievement: A cross-cultural study. Merrill-Palmer Quarterly, 44, 157-172.

Leung, S.O., \& Xu, M.L. (2013). Single-Item measures for subjective academic performance, self-esteem, and socioeconomic status. Journal of Social Service Research, 39, 511-520. doi:10.1080/01488376.2013.794757

Low, S.M., \& Stocker, C. (2005). Family functioning and children's adjustment: Associations among parents' depressed mood, marital hostility, parent-child hostility and children's adjustment. Journal of Family Psychology, 19, 394-403 doi:10.1037/0893-3200.19.3.394

Maccoby, E.E., \& Martin, J.A. (1983). Socialization in the context of the family: Parent-child interaction. In P.H. Mussen (Series Ed.) \& E.M. Hetherington (Vol. Ed.), Handbook of child psychology: Vol. 4. Socialization, personality, and social development (4th ed., pp. 1-101). New York: Wiley.

McLoyd, V.C. (1998). Socioeconomic disadvantage and child development. The American Psychologist, 53, 185-204. doi:10.1037/0003-066X.53.2.185

Noble, K.G., McCandliss, B.D., \& Farah, M.J. (2007). Socioeconomic gradients predict individual differences in neurocognitive abilities. Developmental Science, 10, 464480 .

Papini, D.R., Micka, J.C., \& Barnett, J.K. (1989). Perceptions of intrapsychic and extrapsychic functioning as based of adolescent ego identity statuses. Journal of Adoescent Research, 4, 462-482. doi:10.1177/074355488944005
Perosa, L.M., Perosa, S.L., \& Tam, H.P. (1996). The contribution of family structure and differentiation to identity development in females. Journal of Youth and Adolescence, 5, 817-837.

Quintana, S.M., \& Maxwell, S.E. (1999). Implications of recent developments in structural equation modeling for counseling psychology. Counseling Psychologist, 27, 485527.

R Core Team. (2014). R: A language and environment for statistical computing. Vienna, Austria: R Foundation for Statistical Computing.

Rosseel, Y. (2012). lavaan: An R Package for Structural Equation Modeling. Journal of Statistical Software, 48, 1-36.

Satorra, A., \& Bentler, P.M. (1994). Corrections to test statistics and standard errors in covariance structure analysis. In A. Eye \& C.C. Clogg (Eds.), Latent variables analysis: Applications for developmental research (pp. 399-419). New York: Sage Publications.

Schady, N. (2011). Parents' education, mothers' vocabulary, and cognitive development in early childhood: Longitudinal evidence from Ecuador. American Journal of Public Health, 101, 2299. doi:10.2105/AJPH.2011.300253

Singer, J.A. (1995). Seeing one's self: Locating narrative memory in a framework of personality. Journal of Personality, 63, 429457.

Smits, I., Berzonsky, M.D., Soenens, B., Luyckx, K., Goossens, L., Kunnen, S.E., \& Bosma, H.A. (2008). The Identity Style Inventory - 4. Internal research report. Leuven, Belgium: Catholic University Leuven.

Spera, C. (2005). A review of the relationship among parenting practices, parenting styles, and adolescent school achievement. Educational Psychology Review, 17, 125-146. doi:10.1007/s10648-005-3950-1

Streitmatter, J. (1993). Identity status and identity style: A replication study. Journal of Adolescence. 16, 211-215. doi:10.1006/jabo.1993.1019

Tappan, M.B. (2005). Domination, subordination and the dialogical self: Identity development and the politics of 'ideological becoming'. Culture \& Psychology, 11, 47-75 doi: $10.1177 / 1354067 \times 05050753$

Thomas, M.S.C., Forrester, N.A., \& Ronald, A. (2013). Modeling socioeconomic status effects on language development. Developmental Psychology, 49, 2325-2343. doi:10.1037/a0032301

Thorne, A. (2004). Putting the person into social identity. Human Development, 47, 361-365. doi:10.1159/000081038

Trevatt, D. (2005). Adolescents in mind. Journal of Child Psychotherapy, 31, 221-238. doi:10.1080/00754170500221345

West, S.G., Taylor, A.B., \& Wu, W. (2012). Model fit and model selection in structural equation modeling. In Handbook of structural equation modeling (pp. 209-231). Retrieved from http://books.google.com/books?id=PSiA-7uBmDIC\& pgis $=1$

$\mathrm{Xu}$, C. (2011). A general description of the decade of rule of law in the Macau Special Adminstrative Region. Academic Journal of 'One Country, Two Systems' [(English 
Edition], 1, 170-182. Retrieved August 2014 from http:// www.ipm.edu.mo/images/stories/publication/p_2systems/ academic_eng/issue1/p170.pdf

Yeh, K., \& Bedford, O. (2003). A test of the dual filial piety model. Asian Journal of Social Psychology, 6, 215-228. doi:10.1046/j.1467-839X.2003.00122.x
Zhou, Q., Eisenberg, N., \& Wang, Y. (2004). Chinese children's effortful control and dispositional anger/frustration: relations to parenting styles and children's social functioning. Developmental Psychology, 40, 352-366. doi:10.1037/0012-1649.40.3.352 